\title{
(6) OPEN ACCESS \\ A systematic review of the effects of upper body warm-up on performance and injury
}

\author{
J Matt McCrary, ${ }^{1}$ Bronwen J Ackermann, ${ }^{1}$ Mark Halaki ${ }^{2}$
}

${ }^{1}$ School of Medical Sciences, Sydney Medical School, The University of Sydney, Sydney, New South Wales, Australia ${ }^{2}$ Discipline of Exercise and Sport Science, The University of Sydney, Sydney, New South Wales, Australia

\section{Correspondence to} Dr Bronwen J Ackermann, University of Sydney, Cumberland Campus, PO Box 170, Lidcombe, NSW 1825 Australia; bronwen. ackermann@sydney.edu.au

Accepted 7 January 2015 Published Online First 18 February 2015

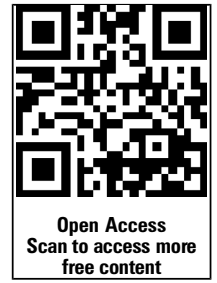

CrossMark

To cite: McCrary JM,

Ackermann BJ, Halaki M. $\mathrm{Br}$

J Sports Med 2015;49:

935-942.

\begin{abstract}
Purpose This systematic review was conducted to identify the impact of upper body warm-up on performance and injury prevention outcomes.
\end{abstract}

Methods Web of Science, MEDLINE, SPORTDiscus, PsycINFO and Cochrane databases were searched using terms related to upper extremity warm-up. Inclusion criteria were English language randomised controlled trials from peer-reviewed journals in which investigation of upper body warm-up on performance and injury prevention outcomes was a primary aim. Included studies were assessed for methodological quality using the PEDro scale. A wide variety of warm-up modes and outcomes precluded meta-analysis except for one group of studies. The majority of warm-ups were assessed as having 'positive', 'neutral', 'negative' or 'specific' effects on outcomes.

Results Thirty-one studies met the inclusion criteria with 21 rated as having 'good' methodological quality. The studies investigated a total of 25 warm-up modes and 43 outcome factors that could be grouped into eight mode and performance outcome categories. No studies of upper body warm-up effects on injury prevention were discovered.

Conclusions Strong research-based evidence was found for the following: high-load dynamic warm-ups enhance power and strength performance; warm-up swings with a standard weight baseball bat are most effective for enhancing bat speed; short-duration static stretching warm-up has no effect on power outcomes; and passive heating/cooling is a largely ineffective warmup mode. A clear knowledge gap in upper body warmup literature is the lack of investigation of injury prevention outcomes.

\section{INTRODUCTION}

Warm-up prior to the start of physical activities is commonplace and lauded by health professionals, ${ }^{1}$ coaches and landmark texts ${ }^{3}$ for its potential for both performance enhancement and injury prevention. The exact mechanisms and outcomes of various warm-up modes, however, are still unclear. A term with broad connotations, warm-up is defined simply by Brukner and $\mathrm{Khan}^{2}$ as being activity that "prepares the body for exercise." There is some disagreement in major sports medical texts regarding acceptable modes of these preparatory activities, especially with regard to whether static stretching is a warm-up activity. ${ }^{2} 3$ For this review, we took a broad view of warm-up modes and defined warm-up as a "protocol specifically undertaken to prepare for the onset of subsequent physical activity.”

Total body and lower extremity warm-up has the potential to both enhance performance and prevent injuries; however, no reviews have been conducted to determine whether and how these effects are replicated in the upper extremity. Considering the different injury mechanisms of common sites of upper and lower extremity injury ${ }^{3} 4$ and differing motor pathways to upper and lower body performance, warm-up effects on the upper extremity need focused investigation. We conducted a systematic review to address questions regarding the optimum upper body warm-up modes for (1) performance enhancement and their physiological correlates across strength, power, endurance, flexibility and accuracy outcomes and (2) injury prevention.

\section{METHODS}

Search criteria are detailed in table 1 , and include all relevant subject headings.

Inclusion criteria were randomised controlled trials (RCTs) published in English language peerreviewed journals in which investigation of the effect of upper body warm-up was a primary aim. An 'upper body warm-up' was defined as “an intervention that targeted the upper extremity and/or core musculature and was designed to prepare the body for subsequent physical activity."

Relevant studies were identified through an initial screening of article titles and abstracts from database and bibliographic search results, followed by a fulltext review of all articles deemed potentially relevant, and a final analysis of their adherence to inclusion criteria. All included studies were assessed according to the PEDro scale, ${ }^{5}$ a systematic tool used to critique RCTs, by two authors (JMM and $\mathrm{MH}$ ), with consultation from the third author (BJA) to resolve disagreements. Papers were given a score from 1 to 10 from a composite of PEDro scale items $2-11 ;^{5}$ item 1 is related to external validity and is not used in the scoring, as per the published PEDro guidelines. Using this scale, studies were classified as having excellent (9-10), good (6-8), fair (4-5) or poor $(<4)$ methodological quality.

Additionally, the primary outcome(s) of included studies were summarised and classified based on the impact of warm-up on these outcome(s). Studies were assessed as having either 'positive', 'neutral' or 'negative' outcomes according to whether the investigated warm-up enhanced, had no effect or degraded, respectively, performance outcome(s). Studies for which positive/neutral/ negative designations were inappropriate (eg, comparisons of two or more modes of warm-up intervention with no control group) were assessed as reporting 'specific effects'. Meta-analysis could only be conducted for four studies that investigated the impact of baseball-specific warm-ups on baseball bat speed, using comparable warm-up conditions. 
Table 1 Search criteria

\begin{tabular}{ll}
\hline Databases & Web of Science (1980-present) \\
searched & MEDLINE (1946-present) \\
& SPORTDiscus (1985-present) \\
& PsycINFO (1806-present) \\
& Cochrane (1966-present) \\
Search terms & "warm up" OR "warm-up" OR "warmup" \\
& AND \\
& "upper extremity" OR "upper limb" OR "back" OR "trunk" \\
& OR "neck" OR "spine" OR "shoulder" OR "elbow" OR \\
& "arm" OR "wrist" OR "hand" OR "forearm" \\
\hline
\end{tabular}

In the absence of consistent quantitative variables that allow for broad meta-analysis, we used the PEDro classification of primary outcomes and qualitative assessment to determine the strength of evidence present for any findings of this review. ${ }^{6} 7$ As such, review results were considered according to four levels of scientific evidence using PEDro and outcome classifications: ${ }^{7}$

- Level 1: Strong research-based evidence: generally consistent findings in multiple high-quality RCTs.

- Level 2: Moderate research-based evidence: generally consistent findings in one high-quality RCT and in one or more low-quality RCTs, or generally consistent findings in multiple low-quality RCTs.

- Level 3: Limited research-based evidence: one RCT (either high quality or low quality) or inconsistent or contradictory evidence in multiple RCTs.

- Level 4: No research-based evidence: no RCTs.

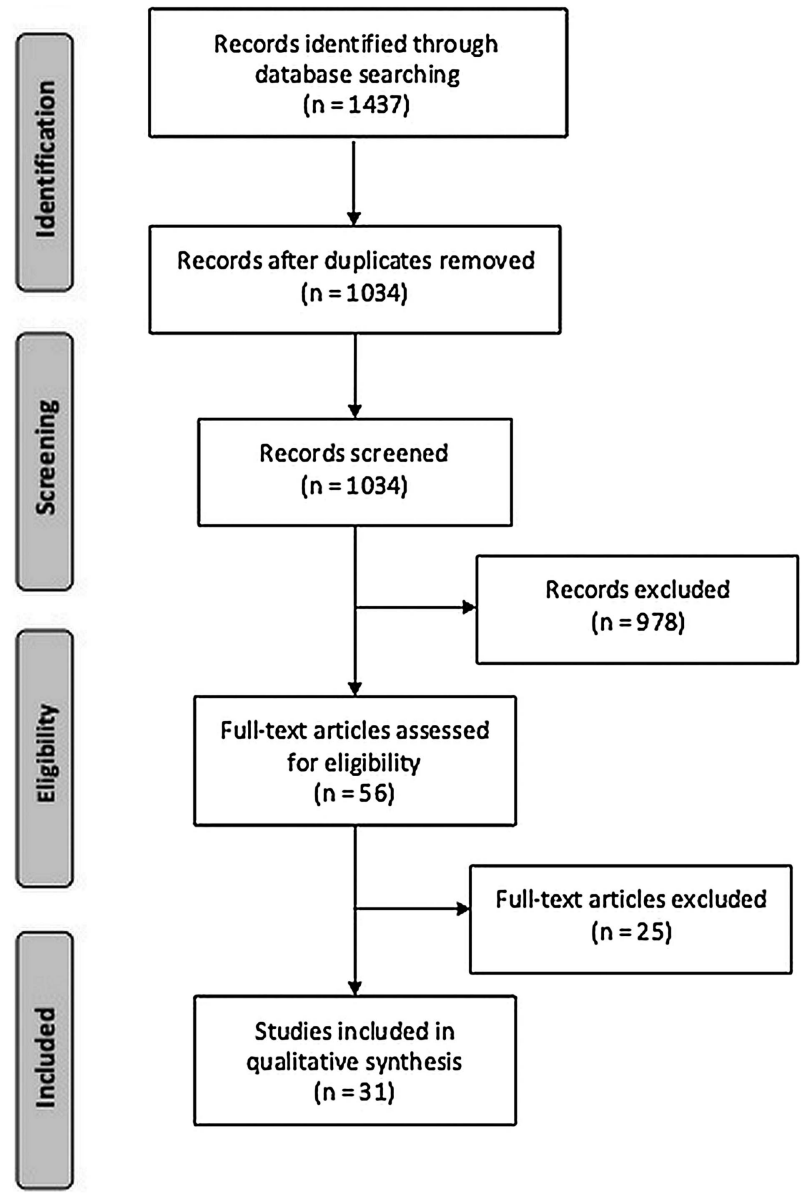

Figure 1 PRISMA flow diagram of literature screening process.
RESULTS

General systematic review statistics

See figure $1 .^{8}$ There were no included articles that investigated the effects of upper body warm-up on injury prevention; all 31 articles studied performance outcomes (see tables 2 and 3).

\section{Included articles—sample size, demographics and population}

The total sample size of all included studies was 628 participants (478 male, 124 female, 26 unreported) with an average sample size of $19 \pm 16$ per study. Sixteen of the included articles investigated warm-up in a population of sport athletes (3 studies of adult athletes, 1 high school, 3 youth, 9 university), with the other 17 studies being investigations of the general population (8 adult, 9 university).

\section{Included articles-classifications of outcomes}

A detailed breakdown of study populations, warm-up modes, outcome factors and outcomes can be found in table 4. Table 5 reports the breakdown of positive/neutral/negative/specific effect outcomes from various warm-up/outcome pairings. 'Warm-up/ outcome' pairing refers to the investigation of the effects of a specific warm-up mode on a single outcome measure. For example, Moran et al's 9 study reported that static stretching had no effect on the golf club head speed or golf club head swing pattern, while dynamic stretching positively impacted both these outcomes. Thus, this study yielded two neutral warm-up/ outcome pairings (static stretching/power and static stretching/ accuracy) and two positive warm-up/outcome pairings (dynamic stretching/power and dynamic stretching/accuracy).

\section{Included articles-meta-analysis}

As seen in figure 2, bat speed is significantly decreased by 'dry swing' warm-ups with either lightweight or heavyweight bats in comparison to the increase observed when using a standard weight bat.

\section{DISCUSSION}

Our results demonstrate that there are no investigations of the effects of upper body warm-up on injury prevention outcomes. The 31 included studies investigated 25 warm-up modes using 43 outcome measures, rendering meta-analysis impractical for all modes except baseball-specific warm-up. To facilitate analysis, we used a simple classification system using 'positive', 'neutral' or 'negative' based on study outcomes in addition to meta-analysis to highlight several observable trends. These trends are summarised below according to the warm-up mode category, along with a classification of the level of evidence present to support these trends. Studies classified as having 'specific effects' on investigated outcomes, as well as a study that investigated the joint effects of two warm-up modes from different categories, ${ }^{10}$ were not included in analyses resulting in level of evidence classifications, but are discussed where relevant.

\section{Dynamic warm-up (levels 1, 2 and 3 evidence)}

The effects of warm-up using dynamic exercises, including steady state, power, body weight and weighted exercises targeting the upper extremities, were investigated in 15 included studies and 56 warm-up/outcome pairings. ${ }^{10-24}$ Exercises classified as being 'dynamic' can be found in table 6. Each upper body dynamic warm-up was also further distinguished with a 'high' or 'low' load classification to analyse the results in the context of the theory of postactivation potentiation, a theory 
Table 2 Breakdown of populations, mode categories, outcome categories and outcome classifications of included studies

\begin{tabular}{|c|c|c|c|c|c|}
\hline Author & $\begin{array}{l}\text { Sample size } \\
\text { (M:F) }\end{array}$ & Population & $\begin{array}{l}\text { Mode category } \\
\text { (incidence) }\end{array}$ & Outcome category (incidence) & $\begin{array}{l}\text { Classification of main } \\
\text { outcome(s) (incidence) }\end{array}$ \\
\hline Bishop et $a 1^{14}$ & $8(4: 4)$ & Young elite kayakers & Dynamic & Physiological (4); power (2) & Specific effects (6) \\
\hline Bishop et al ${ }^{15}$ & $7(7: 0)$ & Elite kayak paddlers & Dynamic & $\begin{array}{l}\text { Physiological (3) } \\
\text { Power (2) }\end{array}$ & $\begin{array}{l}\text { Neutral (3) } \\
\text { Positive (2) }\end{array}$ \\
\hline Brandenburg $^{19}$ & $9(9: 0)$ & University students & Dynamic & Power & Neutral \\
\hline Cé et $a l^{37}$ & $7(2: 5)$ & Recreationally active adults & Static stretch & $\begin{array}{l}\text { Strength } \\
\text { Physiological (4) }\end{array}$ & $\begin{array}{l}\text { Negative } \\
\text { Positive; neutral; negative (2) }\end{array}$ \\
\hline Cochrane et $a l^{22}$ & $12(12: 0)$ & Resistance trained adults & $\begin{array}{l}\text { Vibration } \\
\text { Dynamic }\end{array}$ & $\begin{array}{l}\text { Physiological; power } \\
\text { Physiological; power }\end{array}$ & $\begin{array}{l}\text { Neutral; positive } \\
\text { Neutral; positive }\end{array}$ \\
\hline Demura et $a l^{18}$ & $10(10: 0)$ & Right-handed university students & $\begin{array}{l}\text { Passive heating/ } \\
\text { cooling } \\
\text { Dynamic }\end{array}$ & $\begin{array}{l}\text { Strength; physiological; } \\
\text { Endurance; power } \\
\text { Strength; physiological; endurance }\end{array}$ & $\begin{array}{l}\text { Neutral; positive; neutral; } \\
\text { neutral } \\
\text { Neutral; positive; neutral; } \\
\text { neutral }\end{array}$ \\
\hline DeRenne et $a l^{27}$ & $60(60: 0)$ & High school baseball players & Baseball specific & Power & Specific effects \\
\hline Evans et $a l^{13}$ & $43(16: 27)$ & Untrained university students & $\begin{array}{l}\text { Passive heating/ } \\
\text { cooling } \\
\text { Dynamic }\end{array}$ & $\begin{array}{l}\text { Strength; physiological; flexibility; } \\
\text { passive indicator; DOMS } \\
\text { Strength; physiological; flexibility; } \\
\text { passive indicator; DOMS }\end{array}$ & $\begin{array}{l}\text { Neutral; neutral; positive; } \\
\text { positive; Neutral } \\
\text { Neutral; negative; neutral; } \\
\text { neutral; neutral }\end{array}$ \\
\hline Fradkin et $a l^{10}$ & $20(20: 0)$ & Male golfers & $\begin{array}{l}\text { Dynamic } \\
\text { Static stretch }\end{array}$ & Power & Positive (modes not separated) \\
\hline Franco et $a \beta^{35}$ & $34(34: 0)$ & Active males & $\begin{array}{l}\text { Static stretch } \\
\text { PNF stretch }\end{array}$ & $\begin{array}{l}\text { Endurance (2) } \\
\text { Endurance (2) }\end{array}$ & $\begin{array}{l}\text { Neutral (2) } \\
\text { Negative (2) }\end{array}$ \\
\hline Gelen et $a l^{17}$ & 26 (no data) & Young elite tennis players & $\begin{array}{l}\text { Static stretch } \\
\text { Dynamic }\end{array}$ & $\begin{array}{l}\text { Power } \\
\text { Power }\end{array}$ & $\begin{array}{l}\text { Neutral } \\
\text { Positive }\end{array}$ \\
\hline Haag et $a l^{33}$ & $12(12: 0)$ & University baseball players & Static stretch & Power; accuracy & Neutral (2) \\
\hline Higuchi et a ${ }^{29}$ & $24(24: 0)$ & University baseball field players & $\begin{array}{l}\text { Baseball specific } \\
\text { Isometric } \\
\text { contraction }\end{array}$ & $\begin{array}{l}\text { Power } \\
\text { Power }\end{array}$ & $\begin{array}{l}\text { Specific effects } \\
\text { Positive }\end{array}$ \\
\hline Huang et $a l^{23}$ & $16(16: 0)$ & University baseball players & Dynamic (2) & Power; accuracy & Specific effects (2) \\
\hline Ingham et $a^{20}$ & $10(5: 5)$ & Untrained university students & Dynamic & $\begin{array}{l}\text { Strength (2); physiological; } \\
\text { flexibility; DOMS }\end{array}$ & Positive (5) \\
\hline Kato et $a^{21}$ & $5(5: 0)$ & Healthy Japanese men & Dynamic & Physiological (2) & Positive; neutral \\
\hline Khamwong et al ${ }^{41}$ & $28(28: 0)$ & Untrained male university students & $\begin{array}{l}\text { Passive heating/ } \\
\text { cooling }\end{array}$ & $\begin{array}{l}\text { Strength (2) } \\
\text { Flexibility } \\
\text { Passive indicator (2) } \\
\text { DOMS }\end{array}$ & $\begin{array}{l}\text { Neutral (2) } \\
\text { Positive } \\
\text { Positive; neutral } \\
\text { Neutral }\end{array}$ \\
\hline Knudson et $a \beta^{34}$ & $83(49: 34)$ & Adult tennis players & Static stretch & Power & Neutral \\
\hline Molacek et $a l^{36}$ & $15(15: 0)$ & $\begin{array}{l}\text { Collegiate American Football } \\
\text { players }\end{array}$ & $\begin{array}{l}\text { Passive stretch } \\
\text { PNF stretch }\end{array}$ & Strength & Neutral (2) \\
\hline Montoya et $a^{28}$ & $19(19: 0)$ & Adult recreational baseball players & Baseball specific & Power & Specific effects \\
\hline Moran et al ${ }^{9}$ & $18(18: 0)$ & Male, right hand dominant golfers & $\begin{array}{l}\text { Static stretch } \\
\text { Dynamic stretch }\end{array}$ & $\begin{array}{l}\text { Power; accuracy } \\
\text { Power; accuracy }\end{array}$ & $\begin{array}{l}\text { Neutral (2) } \\
\text { Positive (2) }\end{array}$ \\
\hline Nepocatych et $a l^{24}$ & $10(4: 6)$ & Adult swimmers & $\begin{array}{l}\text { Vibration } \\
\text { Dynamic }\end{array}$ & $\begin{array}{l}\text { Physiological; power (2); Passive } \\
\text { indicator } \\
\text { Physiological; power (2); Passive } \\
\text { indicator }\end{array}$ & $\begin{array}{l}\text { Positive; neutral; neutral; } \\
\text { neutral } \\
\text { Neutral (4) }\end{array}$ \\
\hline $\begin{array}{l}\text { Nosaka and } \\
\text { Clarkson }\end{array}$ & $9(0: 9)$ & Untrained university students & Dynamic (2) & $\begin{array}{l}\text { Strength; physiological; flexibility; } \\
\text { passive indicator; DOMS }\end{array}$ & Positive (10) \\
\hline Nosaka et $\left.a\right|^{43}$ & $20(0: 20)$ & Untrained university students & $\begin{array}{l}\text { Passive heating/ } \\
\text { cooling (2) }\end{array}$ & $\begin{array}{l}\text { Strength } \\
\text { Flexibility } \\
\text { Passive indicator } \\
\text { DOMS }\end{array}$ & $\begin{array}{l}\text { Neutral (2) } \\
\text { Neutral (2) } \\
\text { Positive; neutral } \\
\text { Neutral (2) }\end{array}$ \\
\hline Otsuji et al ${ }^{30}$ & $8(8: 0)$ & $\begin{array}{l}\text { Varsity university baseball/softball } \\
\text { players }\end{array}$ & Baseball specific & Power & Specific effects \\
\hline $\begin{array}{l}\text { Sedgwick and } \\
\text { Whalen }^{44}\end{array}$ & $20(20: 0)$ & Males under age 50 & $\begin{array}{l}\text { Passive heating/ } \\
\text { cooling }\end{array}$ & $\begin{array}{l}\text { Strength } \\
\text { Endurance }\end{array}$ & $\begin{array}{l}\text { Negative } \\
\text { Neutral }\end{array}$ \\
\hline $\begin{array}{l}\text { Southard and } \\
\text { Groomer }\end{array}$ & $10(10: 0)$ & $\begin{array}{l}\text { University students with baseball } \\
\text { experience }\end{array}$ & Baseball specific & Power & Specific effects \\
\hline Symons et $a l^{42}$ & $14(6: 8)$ & Untrained university students & $\begin{array}{l}\text { Passive heating/ } \\
\text { cooling }\end{array}$ & $\begin{array}{l}\text { Strength; physiological; flexibility; } \\
\text { passive indicator; DOMS }\end{array}$ & Neutral (5) \\
\hline Takizawa et al ${ }^{11}$ & $10(10: 0)$ & Untrained university students & Dynamic & $\begin{array}{l}\text { Strength } \\
\text { Physiological (2) } \\
\text { DOMS }\end{array}$ & $\begin{array}{l}\text { Neutral } \\
\text { Positive; neutral } \\
\text { Neutral }\end{array}$ \\
\hline Torres et $a l^{32}$ & $11(11: 0)$ & $\begin{array}{l}\text { University javelin, shot put, } \\
\text { hammer and discus throwers }\end{array}$ & $\begin{array}{l}\text { Static stretch } \\
\text { Dynamic stretch }\end{array}$ & Strength; power & Neutral (16) \\
\hline Wilcox et $a l^{16}$ & $12(12: 0)$ & $\begin{array}{l}\text { University American football or } \\
\text { baseball players }\end{array}$ & Dynamic (2) & Strength & Positive (2) \\
\hline
\end{tabular}


Table 3 Included articles-outcome factors

\begin{tabular}{|c|c|c|}
\hline $\begin{array}{l}\text { Outcome } \\
\text { category }\end{array}$ & Specific outcome factors in category & $\begin{array}{l}\text { Incidence } \\
\text { in included } \\
\text { studies }\end{array}$ \\
\hline \multirow[t]{10}{*}{ Power } & $\begin{array}{l}\text { Peak power (kayak, }{ }_{1}^{27} \text { forearm flexor, }{ }^{29} \text { row, }_{1}{ }^{42} \\
30 \% 1 \text { RM bench press throw, }{ }_{1}^{38} \text { isometric } \\
\text { bench press, }{ }^{38} \text { overhead medicine ball } \\
\text { throw, }^{38} \text { lateral medicine ball throw }{ }^{38} \text { ) }\end{array}$ & 7 \\
\hline & Baseball bat speed ${ }^{27-31}$ & 5 \\
\hline & $\begin{array}{l}\text { Peak acceleration ( } 30 \% \text { of } 1 \mathrm{RM} \text { bench press } \\
\text { throw, isometric bench press, overhead } \\
\text { medicine ball throw, lateral medicine ball } \\
\text { throw) }\end{array}$ & 4 \\
\hline & $\begin{array}{l}\text { Peak displacement ( } 30 \% \text { of } 1 \text { RM bench press } \\
\text { throw, isometric bench press, overhead } \\
\text { medicine ball throw, lateral medicine ball } \\
\text { throw) }\end{array}$ & 4 \\
\hline & $\begin{array}{l}\text { Average power (bench press }{ }^{30} / \text { kayak } \\
\text { paddling }^{1027} \text { ) }\end{array}$ & $3^{141519}$ \\
\hline & Golf club head speed ${ }^{109}$ & 2 \\
\hline & Tennis serve speed ${ }^{17} 34$ & 2 \\
\hline & Throwing speed ${ }^{23} 33$ & 2 \\
\hline & $\begin{array}{l}\text { Sprint performance } \text { (kayak }^{10}, 50 \text {-yard } \\
\text { freestyle }^{32} \text { ) }\end{array}$ & $2^{1424}$ \\
\hline & Total & 31 \\
\hline \multirow[t]{8}{*}{ Strength } & $\begin{array}{l}\text { Immediate maximum isometric } \\
\text { contraction }^{11} 1318203241-44\end{array}$ & 9 \\
\hline & $1 \mathrm{RM}^{162036}$ & 3 \\
\hline & $\begin{array}{l}\text { Peak force }(30 \% \text { of } 1 \mathrm{RM} \text {, overhead and lateral } \\
\text { medicine ball throw) })^{32}\end{array}$ & 3 \\
\hline & Long-term maximum isometric contraction ${ }^{12} 9$ & 2 \\
\hline & Maximum eccentric muscle force ${ }^{43}$ & 1 \\
\hline & Maximum isokinetic contraction ${ }^{42}$ & 1 \\
\hline & Peak torque $\mathrm{e}^{37}$ & 1 \\
\hline & Total & 20 \\
\hline \multirow[t]{15}{*}{ Physiological } & Peak $\mathrm{VO}_{2}{ }^{14} 15$ & 2 \\
\hline & Total $\mathrm{VO}_{2}^{1415}$ & 2 \\
\hline & Accumulated oxygen deficit ${ }^{14} 15$ & 2 \\
\hline & Plasma creatine kinase levels ${ }^{12} 13$ & 2 \\
\hline & Muscle temperature $\mathrm{e}^{111842}$ & 3 \\
\hline & Mean EMG frequency ${ }^{1137}$ & 2 \\
\hline & RMS EMG frequency ${ }^{22} 37$ & 2 \\
\hline & Skin temperature ${ }^{20}$ & 1 \\
\hline & Heart rate ${ }^{24}$ & 1 \\
\hline & Intracellular $\mathrm{pH}^{21}$ & 1 \\
\hline & $\mathrm{Pi} / \mathrm{PCr}$ levels ${ }^{21}$ & 1 \\
\hline & Muscle fibre conduction velocity ${ }^{37}$ & 1 \\
\hline & $1 / 2$ contraction time ${ }^{37}$ & 1 \\
\hline & Total $\mathrm{VCO}_{2}{ }^{15}$ & 1 \\
\hline & Total & 21 \\
\hline \multirow{5}{*}{$\begin{array}{l}\text { Passive } \\
\text { indicators }\end{array}$} & Upper arm circumference ${ }^{12134243}$ & 4 \\
\hline & Pain threshold (cold thermal, pressure) ${ }^{41}$ & 2 \\
\hline & Rating of perceived exertion ${ }^{24}$ & 1 \\
\hline & Stroke count ${ }^{24}$ & 1 \\
\hline & Total & 8 \\
\hline $\begin{array}{l}\text { DOMS }^{11-13} \\
2041-43\end{array}$ & Total & 7 \\
\hline \multirow[t]{3}{*}{ Flexibility } & Elbow ROM ${ }^{12} 13204243$ & 5 \\
\hline & Wrist ROM ${ }^{41}$ & 1 \\
\hline & Total & 6 \\
\hline \multirow[t]{5}{*}{ Endurance } & Bench press endurance ${ }^{35}$ & 1 \\
\hline & Maximum voluntary contraction endurance ${ }^{18}$ & 1 \\
\hline & Dynamic grip endurance ${ }^{44}$ & 1 \\
\hline & Bench press overload volume ${ }^{35}$ & 1 \\
\hline & Total & 4 \\
\hline \multirow[t]{3}{*}{ Accuracy } & Throwing accuracy ${ }^{23} 33$ & 2 \\
\hline & Golf club head swing path ${ }^{9}$ & 1 \\
\hline & Total & 3 \\
\hline
\end{tabular}

DOMS, delayed onset muscle soreness; EMG, Electromyography; RMS, root mean square; ROM, range of motion. proposing that higher load dynamic warm-ups most effectively augment performance through increased intramuscular $\mathrm{Ca}^{2+}$ and cross-bridge cycling. ${ }^{25}$ Sprints, fatiguing isokinetic contractions, plyometrics, steady-state kayak ergometer warm-up at an anaerobic threshold and repeated concentric contractions at a greater than $20 \%$ maximum effort were classified as 'high-load' warm-ups. Investigated 'low-load' dynamic warm-ups were isokinetic warm-ups performed with minimum effort, a lowintensity arm ergometer exercise and repeated gripping of a sponge.

In support of the postactivation potentiation theory, the effects of high-load dynamic warm-ups were overwhelmingly positive, with 16 of 21 mode/outcome pairings reporting a positive outcome. This yielded level 1 evidence of the positive effects of high-load dynamic warm-up on strength ${ }^{12} 1620$ and power $^{15} 1719$ outcomes, as well as level 2 evidence of these positive effects on flexibility ${ }^{12} 20$ and delayed onset muscle soreness (DOMS) ${ }^{12} 20$ outcomes. Level 3 evidence was found regarding physiological ${ }^{12} 1^{15} \quad 20 \quad 21$ and passive indicator ${ }^{12}$ outcomes.

Conversely, the investigated low-load dynamic upper body warm-ups had largely no effect on performance outcomes, as 17 of 27 mode/outcome pairings reported neutral outcomes. Owing to a wide range of investigated outcome categories, no evidence of the effects of low-load dynamic warm-up could be classified as level 1; however, level 2 evidence was found regarding the neutral effects of low-load dynamic warm-up on passive indicator outcomes. ${ }^{12} 1324$ The neutral effects of low-load upper body dynamic warm-up on the following outcomes were classified as being level 3: power, ${ }^{10} 1822{ }^{24}$ strength, ${ }^{11-13}$ endurance, ${ }^{18}$ flexibility ${ }^{12} 13$ and physiological. ${ }^{11-13} 18$ 21-24

Thus, consistent with the theory of postactivation potentiation and the findings of DeRenne, ${ }^{26}$ there is strong evidence that high-load dynamic warm-up enhances upper body power and strength performance, while low-load dynamic warm-ups do not appear to be effective at enhancing any performance variables. Only one study investigated high-load dynamic warm-ups-five reps of 50\%, 75\% and 100\% 5 repetition maximum (RM) bench press ${ }^{19}$ - but did not report positive strength or power outcomes, suggesting that high-load dynamic warm-ups can be recommended for enhancing strength or power outcomes regardless of the specific mode used. Future investigations should focus on determining the optimum load and duration of high-load dynamic warm-up for maximum performance enhancement. Low-load upper body warm-up modes do not appear to have any effect on performance outcomes.

\section{Baseball-specific warm-up (level 1 evidence)}

A subset of dynamic warm-up, studies of warm-up via maximum effort dry swings of baseball bats of various weights have been analysed separately because their identical outcomes (baseball bat speed) enable meta-analysis. Only four of the five studies of baseball-specific warm-up included in this review could be combined for meta-analysis; $;^{27-30}$ the Southard and Groomer $^{31}$ study was excluded from the meta-analysis because bat speed was measured at a different point on the bat and SDs were not reported. The meta-analysis revealed level 1 evidence that dry swing warm-up is a significantly more effective means of enhancing bat speed outcomes when performed with a standard weight bat versus heavyweight and lightweight bats; the Southard and Groomer ${ }^{31}$ study reported similar results to the meta-analysis.

In the context of the theory of postactivation potentiation, the performance decrement resulting from warm-up swings 
Table 4 Included articles-methodological quality (PEDro analysis)

\begin{tabular}{|c|c|c|c|c|c|c|c|c|c|c|c|c|c|}
\hline \multirow[b]{2}{*}{ Study } & \multirow[b]{2}{*}{ Score } & \multirow{2}{*}{$\begin{array}{l}\text { Methodological } \\
\text { quality }\end{array}$} & \multicolumn{11}{|c|}{ PEDro item number } \\
\hline & & & $1^{*}$ & 2 & 3 & 4 & 5 & 6 & 7 & 8 & 9 & 10 & 11 \\
\hline Bishop et $a l^{14}$ & 6 & Good & $\checkmark$ & $\checkmark$ & & $\checkmark$ & & & & $\checkmark$ & $\checkmark$ & $\checkmark$ & $\checkmark$ \\
\hline Bishop et $a l^{15}$ & 6 & Good & $\checkmark$ & $\checkmark$ & & $\checkmark$ & & & & $\checkmark$ & $\checkmark$ & $\checkmark$ & $\checkmark$ \\
\hline Brandenburg ${ }^{19}$ & 6 & Good & $\checkmark$ & $\checkmark$ & & $\checkmark$ & & & & $\checkmark$ & $\checkmark$ & $\checkmark$ & $\checkmark$ \\
\hline Cé et $a l^{37}$ & 6 & Good & $\checkmark$ & $\checkmark$ & & $\checkmark$ & & & & $\checkmark$ & $\checkmark$ & $\checkmark$ & $\checkmark$ \\
\hline Cochrane et $a^{22}$ & 6 & Good & $\checkmark$ & $\checkmark$ & & $\checkmark$ & & & & $\checkmark$ & $\checkmark$ & $\checkmark$ & $\checkmark$ \\
\hline Demura et $a l^{18}$ & 5 & Fair & $\checkmark$ & & & $\checkmark$ & & & & $\checkmark$ & $\checkmark$ & $\checkmark$ & $\checkmark$ \\
\hline DeRenne et $a l^{27}$ & 6 & Good & $\checkmark$ & $\checkmark$ & & $\checkmark$ & & & & $\checkmark$ & $\checkmark$ & $\checkmark$ & $\checkmark$ \\
\hline Evans et $a l^{13}$ & 6 & Good & $\checkmark$ & $\checkmark$ & & $\checkmark$ & & & & $\checkmark$ & $\checkmark$ & $\checkmark$ & $\checkmark$ \\
\hline Fradkinet $a l^{10}$ & 5 & Fair & $\checkmark$ & $\checkmark$ & & $\checkmark$ & & & & $\checkmark$ & $\checkmark$ & $\checkmark$ & \\
\hline Franco et $\left.a\right|^{35}$ & 6 & Good & $\checkmark$ & $\checkmark$ & & $\checkmark$ & & & & $\checkmark$ & $\checkmark$ & $\checkmark$ & $\checkmark$ \\
\hline Gelen et $a l^{17}$ & 6 & Good & $\checkmark$ & $\checkmark$ & & $\checkmark$ & & & & $\checkmark$ & $\checkmark$ & $\checkmark$ & $\checkmark$ \\
\hline Haag et $a l^{33}$ & 5 & Fair & $\checkmark$ & & & $\checkmark$ & & & & $\checkmark$ & $\checkmark$ & $\checkmark$ & $\checkmark$ \\
\hline Higuchi et al $l^{29}$ & 5 & Fair & $\checkmark$ & & & $\checkmark$ & & & & $\checkmark$ & $\checkmark$ & $\checkmark$ & $\checkmark$ \\
\hline Huang et $a l^{23}$ & 7 & Good & $\checkmark$ & $\checkmark$ & & $\checkmark$ & & & $\checkmark$ & $\checkmark$ & $\checkmark$ & $\checkmark$ & $\checkmark$ \\
\hline Ingham et $a^{20}$ & 6 & Good & $\checkmark$ & $\checkmark$ & & $\checkmark$ & & & & $\checkmark$ & $\checkmark$ & $\checkmark$ & $\checkmark$ \\
\hline Kato et $a^{21}$ & 6 & Good & $\checkmark$ & $\checkmark$ & & $\checkmark$ & & & & $\checkmark$ & $\checkmark$ & $\checkmark$ & $\checkmark$ \\
\hline Khamwong et $a l^{41}$ & 6 & Good & $\checkmark$ & $\checkmark$ & & $\checkmark$ & & & & $\checkmark$ & $\checkmark$ & $\checkmark$ & $\checkmark$ \\
\hline Knudson et $a l^{34}$ & 4 & Fair & $\checkmark$ & & & & & & & $\checkmark$ & $\checkmark$ & $\checkmark$ & $\checkmark$ \\
\hline Molacek et $a l^{36}$ & 6 & Good & $\checkmark$ & $\checkmark$ & & $\checkmark$ & & & & $\checkmark$ & $\checkmark$ & $\checkmark$ & $\checkmark$ \\
\hline Montoya et $a l^{28}$ & 6 & Good & $\checkmark$ & $\checkmark$ & & $\checkmark$ & & & & $\checkmark$ & $\checkmark$ & $\checkmark$ & $\checkmark$ \\
\hline Moran et $a l^{9}$ & 5 & Fair & $\checkmark$ & & & $\checkmark$ & & & & $\checkmark$ & $\checkmark$ & $\checkmark$ & $\checkmark$ \\
\hline Nepocatych et al ${ }^{24}$ & 5 & Fair & $\checkmark$ & & & $\checkmark$ & & & & $\checkmark$ & $\checkmark$ & $\checkmark$ & $\checkmark$ \\
\hline Nosaka and Clarkson ${ }^{12}$ & 5 & Fair & $\checkmark$ & & & $\checkmark$ & & & & $\checkmark$ & $\checkmark$ & $\checkmark$ & $\checkmark$ \\
\hline Nosaka et a $/^{43}$ & 6 & Good & $\checkmark$ & $\checkmark$ & & $\checkmark$ & & & & $\checkmark$ & $\checkmark$ & $\checkmark$ & $\checkmark$ \\
\hline Otsuji et $a l^{30}$ & 6 & Good & $\checkmark$ & & & $\checkmark$ & & & $\checkmark$ & $\checkmark$ & $\checkmark$ & $\checkmark$ & $\checkmark$ \\
\hline Sedgwick and Whalen ${ }^{44}$ & 4 & Fair & $\checkmark$ & & & & & & & $\checkmark$ & $\checkmark$ & $\checkmark$ & $\checkmark$ \\
\hline Southard and Groomer ${ }^{31}$ & 4 & Fair & $\checkmark$ & $\checkmark$ & & & & & & $\checkmark$ & $\checkmark$ & $\checkmark$ & \\
\hline Symons et $a l^{42}$ & 7 & Good & $\checkmark$ & $\checkmark$ & & $\checkmark$ & $\checkmark$ & & & $\checkmark$ & $\checkmark$ & $\checkmark$ & $\checkmark$ \\
\hline Takizawa et al ${ }^{11}$ & 6 & Good & $\checkmark$ & $\checkmark$ & & $\checkmark$ & & & & $\checkmark$ & $\checkmark$ & $\checkmark$ & $\checkmark$ \\
\hline Torres et $a l^{32}$ & 6 & Good & $\checkmark$ & $\checkmark$ & & $\checkmark$ & & & & $\checkmark$ & $\checkmark$ & $\checkmark$ & $\checkmark$ \\
\hline Wilcox et $a l^{16}$ & 6 & Good & $\checkmark$ & $\checkmark$ & & $\checkmark$ & & & & $\checkmark$ & $\checkmark$ & $\checkmark$ & $\checkmark$ \\
\hline
\end{tabular}

${ }^{*}$ Not included in methodological quality scoring

with a lightweight bat appears consistent; however, the same result following warm-up swings with a heavyweight bat appears to be contradictory to postactivation potentiation. One possible explanation is that the use of a weighted bat during warm-up may have resulted in biomechanical adaptations that were not ideal for maximising bat speed with a normal weight bat, as supported by the Southard and Groomer ${ }^{31}$ study. These authors noted that the weighted bat used in their study had a significantly different moment of inertia than the normal weight bat, and that the swing mechanics for the normal bat were significantly altered following a weighted bat warm-up. ${ }^{31}$ These alterations in mechanics appear to also supersede any perceptual benefits; a separate study found that participants perceived their bat speed to be significantly faster following weighted warm-up swings, when the actual bat speed was significantly reduced. ${ }^{30}$

The decrease in bat speed reported following the weighted bat warm-up may also have been influenced by the extremely short duration of each bat swing or the mandated rest period between swings in each study (at least $5 \mathrm{~s}$ ); 4-5 isolated dry swings with a heavy bat may not work the musculature enough to produce the anticipated post activation potentiation from this higher loading warm-up. The threshold of muscle activity necessary to produce a postactivation potentiation response, however, does not appear to be extremely high, given that Higuchi et $a l^{29}$ reported that five sets of $5 \mathrm{~s}$ maximal isometric contractions in a batting stance, separated by a $5 \mathrm{~s}$ rest, significantly increased bat speed.

Further high-quality investigation is required to explain why dry swing warm-ups with a standard weight bat are most effective. Specifically, this research should include highlighted factors such as determining the minimum duration of muscle activity necessary to elicit a postactivation potentiation response, the relationships between perceived and observed performance outcomes, and the importance of task-specific equipment in warm-up to enhance performance outcomes.

\section{Maximum isometric contraction (level 3 evidence)}

Only one included study investigated the effects of maximum isometric contraction warm-up and found that $5 \mathrm{~s}$ of maximum isometric contraction warm-up significantly increased baseball bat speed. ${ }^{29}$ Given that maximum isometric contraction is a high-load, albeit static, warm-up mode, this isolated result makes sense in the context of postactivation potentiation but needs to be validated by further research.

\section{Stretching-static (levels 1 and 3 evidence)}

Static stretching was found to be a largely ineffective method for performance enhancement. Despite the fact that 25 of 31 
Table 5 Warm-up/outcome pairings, organised by category

\begin{tabular}{|c|c|c|c|c|c|c|}
\hline Mode category & $\begin{array}{l}\text { Outcome } \\
\text { category }\end{array}$ & $\begin{array}{l}\text { Warm-up/outcome } \\
\text { pairings }\end{array}$ & $\begin{array}{l}\text { Positive } \\
\text { outcomes }\end{array}$ & $\begin{array}{l}\text { Neutral } \\
\text { outcomes }\end{array}$ & $\begin{array}{l}\text { Negative } \\
\text { outcomes }\end{array}$ & $\begin{array}{l}\text { Specific } \\
\text { effects }\end{array}$ \\
\hline & Physiological & $19^{11-22} 24$ & $6^{1112182021}$ & $8^{1115212224}$ & $1^{13}$ & $4^{14}$ \\
\hline & Power & $11^{10} 141517-1922-24$ & $5^{10151722}$ & $3^{181924}$ & - & $3^{1423}$ \\
\hline & Strength & $9^{11-1316182042}$ & $6^{121620}$ & $3^{111318}$ & - & - \\
\hline \multirow[t]{6}{*}{ Dynamic } & DOMS & $5^{11-1320}$ & $3^{1220}$ & $2^{1113}$ & - & - \\
\hline & Flexibility & $4^{121320}$ & $3^{1220}$ & $1^{13}$ & - & - \\
\hline & Passive indicators & $5^{121324}$ & $2^{12}$ & $3^{1324}$ & - & - \\
\hline & Endurance & $1^{18}$ & - & $1^{18}$ & - & - \\
\hline & Accuracy & $1^{23}$ & - & - & - & $1^{23}$ \\
\hline & Total & 56 & 25 & 22 & 1 & 8 \\
\hline \multirow[t]{2}{*}{ Baseball-specific warm-up } & Power & $5^{27-31}$ & - & - & - & $5^{27-31}$ \\
\hline & Total & 5 & 0 & 0 & 0 & 5 \\
\hline \multirow{4}{*}{$\begin{array}{l}\text { Maximum isometric } \\
\text { contraction }\end{array}$} & Power & $1^{29}$ & $1^{29}$ & - & - & - \\
\hline & Total & 1 & 1 & 0 & 0 & 0 \\
\hline & Power & $17^{910} 1732-34$ & $1^{10}$ & $16^{9} 1732-34$ & - & - \\
\hline & Physiological & $4^{37}$ & $1^{37}$ & - & $3^{37}$ & - \\
\hline \multirow[t]{5}{*}{ Static stretching } & Strength & $6^{32} 3637$ & - & $5^{32} 3637$ & $1^{37}$ & - \\
\hline & Endurance & $2^{35}$ & - & $2^{35}$ & - & - \\
\hline & Accuracy & $2^{933}$ & - & $2^{933}$ & - & - \\
\hline & Total & 31 & 2 & 25 & 4 & 0 \\
\hline & Power & $13^{329}$ & $12^{9}$ & $1^{32}$ & - & - \\
\hline \multirow[t]{3}{*}{ Dynamic stretching } & Accuracy & $1^{9}$ & $1^{9}$ & - & - & - \\
\hline & Strength & $4^{1832}$ & - & $4^{32}$ & - & - \\
\hline & Total & 18 & 13 & 5 & 0 & 0 \\
\hline \multirow[t]{6}{*}{ PNF stretching } & Endurance & $2^{35}$ & - & - & $2^{35}$ & - \\
\hline & Strength & $1^{36}$ & - & $1^{36}$ & - & - \\
\hline & Total & 3 & 0 & 1 & 2 & 0 \\
\hline & Strength & $12^{131841-44}$ & - & $11^{131841-44}$ & $1^{44}$ & - \\
\hline & Passive indicators & $6^{1341-43}$ & $2^{1341}$ & $4^{41-43}$ & - & - \\
\hline & DOMS & $5^{1341-43}$ & - & $5^{1341-43}$ & - & - \\
\hline \multirow[t]{5}{*}{ Passive heating/cooling } & Flexibility & $5^{1341-43}$ & $3^{134143}$ & $2^{4243}$ & - & - \\
\hline & Physiological & $3^{131842}$ & $1^{18}$ & $2^{1342}$ & - & - \\
\hline & Endurance & $2^{1844}$ & - & $2^{1844}$ & - & - \\
\hline & Power & $1^{18}$ & - & $1^{18}$ & - & - \\
\hline & Total & 34 & 6 & 27 & 1 & 0 \\
\hline \multirow[t]{4}{*}{ Vibration } & Power & $3^{2224}$ & $1^{22}$ & $2^{24}$ & - & - \\
\hline & Physiological & $2^{2224}$ & $1^{24}$ & $1^{22}$ & - & - \\
\hline & Passive indicators & $2^{24}$ & - & $2^{24}$ & - & - \\
\hline & Total & 7 & 2 & 5 & 0 & 0 \\
\hline
\end{tabular}

DOMS, delayed onset muscle soreness.

Chart Area Otsuji et al. (2002)

Montoya et al. (2009)

DeRenne et al. (1992)

Higuchi et al. (2013)

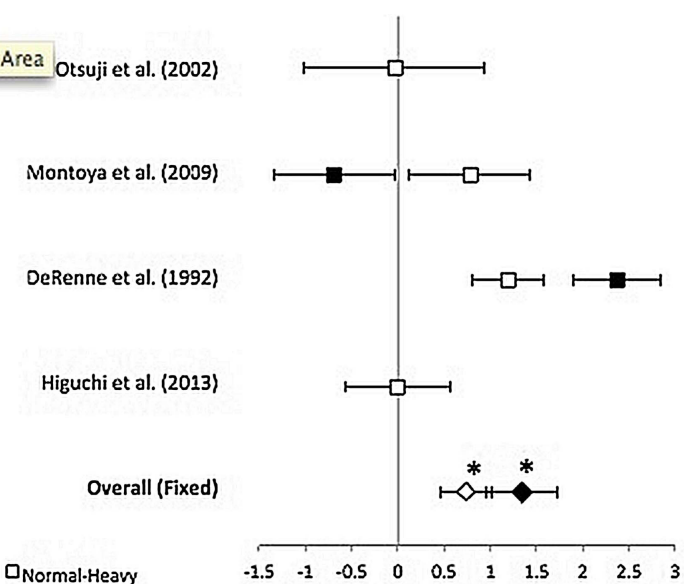

Q Normal-Heavy

Normal-Light
Standard Difference in the means $(\mathrm{m} / \mathrm{s}) \quad * p<.05$

Figure 2 Bat speed.

warm-up/outcome pairings reported neutral outcomes, ${ }^{9} 17$ 32-37 the review only yielded level 1 evidence regarding the neutral effects of static stretch warm-up on power outcomes. ${ }^{9} 17 \quad 32-34$ Level 3 evidence was discovered for the following effects: static stretch warm-up had no effect on strength, ${ }^{32} 3637$ accuracy $^{9} 33$ and endurance ${ }^{35}$ outcomes; and static stretch warm-up negatively impacted physiological outcomes. ${ }^{37}$

Earlier reviews of total body and lower extremity static stretch warm-up proposed a $60 \mathrm{~s}^{38}$ or $90 \mathrm{~s}^{39}$ threshold for static stretch duration without performance decrement. As such, included investigations of upper body static stretch warm-up were grouped by stretch duration into either short $(\leq 60 \mathrm{~s})^{17}{ }^{32-}$ 35 or long $(>90 \mathrm{~s})^{93637}$ groups for further analysis; no studies investigated stretches lasting between 60 and 90 s. Even with these duration groupings, outcomes and levels of evidence for investigated outcomes remained unchanged. The notable exception was that long-duration static stretch was found to have no effect on power outcomes with only level 3 evidence due to the presence of only one investigation of this warm-up/outcome 
Table 6 Included articles-warm-up mode

\begin{tabular}{|c|c|c|}
\hline Mode category & Specific modes in category & $\begin{array}{l}\text { Incidence } \\
\text { in included } \\
\text { studies }\end{array}$ \\
\hline \multirow[t]{15}{*}{ Dynamic exercises } & $\begin{array}{l}\text { Isokinetic contractions at minimal } \\
\text { effort }^{11-13}\end{array}$ & 3 \\
\hline & $\begin{array}{l}\text { Kayak ergometer (steady state or steady } \\
\text { state with sprints) }\end{array}$ & 2 \\
\hline & Upper body plyometrics ${ }^{16} 17$ & 2 \\
\hline & $\begin{array}{l}\text { Repeated forearm contractions around } \\
\text { sponge }^{18}\end{array}$ & 1 \\
\hline & $\begin{array}{l}\text { Isometric contractions at fatiguing } \\
\text { effort }^{12}\end{array}$ & 1 \\
\hline & Percentage of 5RM bench press ${ }^{19}$ & 1 \\
\hline & Percentage of 1 RM biceps curls ${ }^{20}$ & 1 \\
\hline & Percentage of wrist flexion $\mathrm{MVC}^{21}$ & 1 \\
\hline & Arm ergometer ${ }^{22}$ & 1 \\
\hline & Sling exercise ${ }^{23}$ & 1 \\
\hline & Free weights/resistive tubing ${ }^{23}$ & 1 \\
\hline & Medicine ball throws ${ }^{16}$ & 1 \\
\hline & Competition swimming warm-up ${ }^{24}$ & 1 \\
\hline & $\begin{array}{l}\text { Body weight exercises (arm circles, } \\
\text { windmills, trunk twists) }\end{array}$ & 1 \\
\hline & Total & 18 \\
\hline \multirow{4}{*}{$\begin{array}{l}\text { Baseball-specific } \\
\text { warm-up }\end{array}$} & Dry swings with weighted bat ${ }^{27-31}$ & 5 \\
\hline & Dry swings with unweighted bat ${ }^{27-31}$ & 5 \\
\hline & Dry swings with lightweight bat ${ }^{27} 2831$ & 3 \\
\hline & Total & 13 \\
\hline \multirow[t]{3}{*}{ Passive heating/cooling } & Passive heating ${ }^{12} 131841-44$ & 7 \\
\hline & Passive cooling ${ }^{43}$ & 1 \\
\hline & Total & 8 \\
\hline \multirow[t]{2}{*}{ Static stretching } & Self-administered ${ }^{9} 17$ 32-34 & 5 \\
\hline & $\begin{array}{l}\text { Passive-stretch held by researcher/ } \\
\text { therapist }{ }^{35-37}\end{array}$ & 3 \\
\hline Dynamic stretching & Dynamic stretching ${ }^{32} 9$ & 2 \\
\hline PNF stretching & PNF stretching 3536 & 2 \\
\hline Vibration & Vibration 2224 & 2 \\
\hline $\begin{array}{l}\text { Maximum isometric } \\
\text { contraction }\end{array}$ & Maximum isometric contraction ${ }^{29}$ & 1 \\
\hline
\end{tabular}

pairing. ${ }^{9}$ The neutral effects of short-duration static stretching on power outcomes were classified as level 1 evidence. ${ }^{17}$ 32-34

Thus, the only strong evidence present in the literature is that short-duration upper body static stretch warm-ups can be conducted without decreasing power, ${ }^{17}{ }^{32-34}$ a finding that is consistent with previous reviews of total and lower body static stretch warm-up. ${ }^{38} 39$ Further investigation is necessary to confirm the level 3 finding that long-duration static stretching can also be performed without power decrement, and also to clarify the effects of both long-duration and short-duration static stretching on strength, accuracy, physiological and endurance outcomes.

\section{Stretching-dynamic (level 3 evidence)}

Only two included ${ }^{329}$ studies investigated the effects of dynamic stretching warm-up on four total outcomes, with Torres et al ${ }^{32}$ reporting that dynamic stretching had no effect on a variety of upper body force and power measures (see table 3), and Moran et $a l^{9}$ finding that dynamic stretching increased golf club head speed and accuracy. Given these mixed results, the evidence is classified as level 3. The absence of reported negative outcomes suggests that upper body dynamic stretching warm-ups can most likely be performed without negative effects. The benefits of upper body dynamic stretching warm-up, however, need to be clarified with further investigation.
Stretching-proprioceptive neuromuscular facilitation (level 3 evidence)

The two included studies ${ }^{35} 36$ of upper body proprioceptive neuromuscular facilitation (PNF) stretching warm-ups found that PNF stretching had no effect on strength outcomes ${ }^{36}$ but negatively impacted endurance outcomes. ${ }^{35}$ Evidence is classified as level 3. No studies investigated the effects of upper body PNF stretching warm-up on flexibility outcomes-the main reported benefit of PNF stretching ${ }^{40}$ - so the utility of this warm-up mode in the upper body remains unclear (table 6).

\section{Passive heating/cooling (levels 1, 2 and 3 evidence)}

Passive heating and/or cooling appear to have extremely limited use as a warm-up mode, with the only positive outcome being that passive heating warm-ups positively affect flexibility outcomes for up to 8 days following fatiguing eccentric exercise (level 1 evidence). ${ }^{12} 13 \quad 41-43$ The acute effects of passive heating warm-up were not investigated. Level 1 evidence also supported the conclusion that passive heating or cooling warm-ups do not affect DOMS, ${ }^{12} 13$ 41-43 or strength $^{12} 1318$ 41-43 outcomes. Level 2 evidence shows that passive heating warm-ups do not have any impact on endurance outcomes, ${ }^{18}{ }^{44}$ and level 3 evidence suggests that neither passive heating nor passive cooling warm-ups impact passive indicator, ${ }^{13}{ }^{41-43}$ physiological $^{131842}$ or power $^{18}$ outcomes. Thus, the only notable conclusion that can be presented regarding upper body passive heating and/or cooling warm-up is that passively heating muscles before a fatiguing eccentric exercise is likely to significantly minimise losses in flexibility in the days following the fatiguing exercise. Future investigations should focus on any acute flexibility benefits from upper body passive heating warm-up; however, the benefits of this warm-up on performance outcomes seem to be limited.

\section{Vibration (levels 2 and 3 evidence)}

Upper body vibration warm-ups were investigated in two included studies and six warm-up/outcome pairings, ${ }^{22} 24$ with limited results. Vibration warm-up was found, with level 2 evidence, to have no effect on physiological outcomes 22 and, supported by level 3 evidence, has no effect on passive indicator outcomes $^{24}$ and mixed effects on power outcomes. ${ }^{22}{ }^{24}$ Of note is the fact that vibration warm-up was performed with the participant lying prone on a bench in both studies of power outcomes, with prone row power experiencing significant postwarm-up improvements, ${ }^{22}$ but not 50 yard freestyle swim time. ${ }^{24}$ As such, there may be a level of similarity between vibration warm-up mode and activity necessary to achieve performance benefits.

\section{Injury prevention (level 4 evidence)}

We found no studies of upper body warm-up with injury prevention outcomes. Thus, while warming-up for injury prevention purposes is popular, this practice is not yet supported by evidence for upper body activities. The American Academy of Orthopaedic Surgeons notes that 5 of the 10 most common orthopaedic injuries occur in the upper extremity ${ }^{45}$ and it recommends, along with many others, ${ }^{1-3}$ warm-up as an important part of an injury prevention plan. Thus, there is a clear need for high-quality scientific research evidence to support these warm-up recommendations currently based only on theory, sporting experience and anecdotal evidence.

The current literature on upper body warm-up covers a wide range of warm-up modes and performance outcomes (25 
modes, 43 outcomes). Several trends may guide future research and clinical practice.

- For the most part, results were consistent with findings of previous reviews of total and lower body warm-up.

- We found strong evidence that high-load dynamic upper body warm-ups enhance both strength and power outcomes. There may, however, be a minimum duration of muscle loading (exceeding 4-5 dry swings of a weighted baseball bat) for these warm-ups to have the desired effect. Task-specific warm-ups were effective when used in baseball; however, no evidence exists to support task-specific warm-up in other domains.

- Upper body static stretching warm-ups of a duration $\leq 60 \mathrm{~s}$ had no impact on power outcomes. Long-duration (>90 s) static stretching can also be conducted without performance decrement, but more data are needed to validate this finding.

- Passive heating/cooling warm-ups do not appear to have any significant acute performance effects, although flexibility in the days following fatiguing eccentric exercise can be enhanced with this mode of warm-up.

- Additional studies are needed to clarify the effects of upper body maximum isometric contraction, dynamic and PNF stretching, and vibration training warm-ups.

- Further investigation is especially needed across all warm-up modes to validate recommendations of using warm-up as a means of injury prevention.

\section{Clinical applications (author commentary)}

Based on the evidence of this review, an optimum upper body warm-up regimen should contain a combination of high-load dynamic warm-ups to enhance performance and short-duration (<60 s) static stretching for flexibility gains. Assuming at least a general application of previously noted correlations between flexibility and injury risk ${ }^{46} 47$ to the upper body, these flexibility gains may have some preventative effects. A simple and effective general shoulder warm-up, for example, could involve a combination of high-intensity activities to induce postactivation potentiation in all three planes of movement, along with static stretching of the pectorals, trapezius, latissimus dorsi and deltoids to maintain flexibility. The high-load exercises and static stretches to be used in sport-specific warm-ups should closely mirror the required movements and mechanics of the sport (eg, throwing, batting) rather than using only a general protocol. As noted previously, further research is necessary to validate the efficacy task-specific warm-up in domains beyond baseball and static stretching for injury prevention.

\section{CONCLUSIONS}

Our systematic review found strong evidence for the following: high-load dynamic warm-ups enhance power and strength

\section{What this study adds?}

- A common recommendation of using warm-up exercises to prevent upper body injuries is not supported by any investigations.

- There is strong evidence that dynamic, high-load upper body warm-ups effectively enhance strength and power outcomes.

- Using static stretching in upper body warm-ups that last less than $60 \mathrm{~s}$ can be prescribed to enhance flexibility without impacting power and strength outcomes. performance; warm-up swings with a standard weight baseball bat are most effective for enhancing bat speed; short-duration static stretching warm-up has no effect on power outcomes; and passive heating/cooling is a largely ineffective warm-up mode. Maximum isometric contraction may also enhance performance variables. Dynamic stretching and long-duration upper body static stretching warm-up may be able to be performed without adverse performance effects. A striking knowledge gap in upper body warm-up literature is the lack of investigation of injury prevention outcomes.

Contributors All three authors collaborated to provide the concept and focus for this review, have approved the manuscript, and agreed to be accountable for all aspects of the work. JMM performed the literature searches and selected the articles to be included in this systematic review. He also synthesised, analysed and summarised the positive/neutral/negative/specific outcomes of included articles, scored the included articles according to the PEDro scale, and drafted the introduction, methods, results, and the majority of the discussion and conclusion. BJA drafted portions of the discussion and conclusion, made significant revisions to the entire manuscript, and served as a consultant for the PEDro scoring and analysis of outcomes. MH scored the included articles according to the PEDro scale, performed the meta-analysis, made significant revisions to the entire manuscript, and served as a consultant regarding the analysis of outcomes.

Funding JMM was supported by the Australian-American Fulbright Commission.

\section{Competing interests None.}

Provenance and peer review Not commissioned; externally peer reviewed.

Open Access This is an Open Access article distributed in accordance with the Creative Commons Attribution Non Commercial (CC BY-NC 4.0) license, which permits others to distribute, remix, adapt, build upon this work non-commercially, and license their derivative works on different terms, provided the original work is properly cited and the use is non-commercial. See: http://creativecommons.org/ licenses/by-nc/4.0/

\section{REFERENCES}

1 Brooks JHM, Erith SJ. Warm-up programmes in sport. BMJ 2008;337:61-2.

2 Brukner $P$, Khan $K$, Bahr R. Principles of injury prevention. In: Brukner $P$, Khan $K$, eds. Brukner \& Khan's clinical sports medicine. Sydney: McGraw-Hill, 2012:81.

3 Delee J, Drez D, Miller MD. Delee \& Drez's orthopaedic sports medicine: principles and practice. Philadelphia: Saunders/Elsevier, 2010.

4 Boden BP, Dean GS, Feagin JJA, et al. Mechanisms of anterior cruciate ligament injury. Orthopedics 2000;23:573-8

5 PEDro. PEDro Scale. http://www.pedro.org.au/english/downloads/pedroscale/

6 Reid SA, Rivett DA. Manual therapy treatment of cervicogenic dizziness: a systematic review. Man Ther 2005;10:4-13.

7 van Tulder MW, Assendelft WJ, Koes BW, et al. Method guidelines for systematic reviews in the Cochrane Collaboration Back Review Group for Spinal Disorders. Spine 1997;22:2323-30.

8 PRISMA. Transparent reporting of systematic reviews and meta-analyses. Secondary transparent reporting of systematic reviews and meta-analyses. http://www. prisma-statement.org

9 Moran KA, McGrath T, Marshall BM, et al. Dynamic stretching and golf swing performance. Int J Sports Med 2009;30:113-18.

10 Fradkin AJ, Sherman CA, Finch CF. Improving golf performance with a warm up conditioning programme. Br J Sports Med 2004;38:762-5.

11 Takizawa K, Soma T, Nosaka K, et al. Effect of warm-up exercise on delayed-onset muscle soreness. Eur J Sport Sci 2011;12:1-7.

12 Nosaka K, Clarkson PM. Influence of previous concentric exercise on eccentric exercise-induced muscle damage. J Sports Sci 1997;15:477-83.

13 Evans RK, Knight KL, Draper DO, et al. Effects of warm-up before eccentric exercise on indirect markers of muscle damage. Med Sci Sports Exerc 2002;34:1892.

14 Bishop D, Bonetti D, Dawson B. The effect of three different warm-up intensities on kayak ergometer performance. Med Sci Sports Exerc 2001;33:1026-32.

15 Bishop D, Bonetti D, Spencer M. The effect of an intermittent, high-intensity warm-up on supramaximal kayak ergometer performance. J Sports Sci 2003;21:13-20.

16 Wilcox J, Larson R, Brochu KM, et al. Acute explosive-force movements enhance bench-press performance in athletic men. Int J Sports Physiol Perform 2006;1:261-9.

17 Gelen E, Dede M, Bingul BM, et al. Acute effects of static stretching, dynamic exercises, and high volume upper extremity plyometric activity on tennis serve performance. J Sports Sci Med 2012;11:600-5.

18 Demura T, Demura S, Aoki H, et al. Effect of linear polarized near-infrared light irradiation and light exercise on muscle performance. J Physiol Anthropol 2011;30:91-6. 
19 Brandenburg JP. The acute effects of prior dynamic resistance exercise using different loads on subsequent upper-body explosive performance in resistance-trained men. J Strength Cond Res 2005;19:427-32.

20 Ingham SA, van Someren KA, Howatson G. Effect of a concentric warm-up exercise on eccentrically induced soreness and loss of function of the elbow flexor muscles. J Sports Sci 2010;28:1377-82.

21 Kato $\mathrm{Y}$, Ikata $\mathrm{T}$, Takai $\mathrm{H}$, et al. Effects of specific warm-up at various intensities on energy metabolism during subsequent exercise. I Sports Med Phys Fitness 2000;40:126-30

22 Cochrane DJ, Stannard SR, Walmsely A, et al. The acute effect of vibration exercise on concentric muscular characteristics. I Sci Med Sport 2008;11:527-34.

23 Huang JS, Pietrosimone BG, Ingersoll $C D$, et al. Sling exercise and traditional warm-up have similar effects on the velocity and accuracy of throwing. I Strength Cond Res 2011;25:1673-9.

24 Nepocatych S, Bishop PA, Balilionis G, et al. Acute effect of upper-body vibration on performance in master swimmers. J Strength Cond Res 2010;24:3396-403.

25 Sale DG. Postactivation potentiation: role in human performance. Exerc Sport Sci Rev 2002;30:138-43.

26 DeRenne C. Effects of postactivation potentiation warm-up in male and female sport performances: a brief review. Strength Cond J 2010;32:58-64.

27 DeRenne C, Ho KW, Hetzler RK, et al. Effects of warm up with various weighted implements on baseball bat swing velocity. J Strength Cond Res 1992;6:214-18.

28 Montoya BS, Brown LE, Coburn JW, et al. Effect of warm-up with different weighted bats on normal baseball bat velocity. J Strength Cond Res 2009;23:1566-9.

29 Higuchi T, Nagami T, Mizuguchi N, et al. The acute and chronic effects of isometric contraction conditioning on baseball bat velocity. J Strength Cond Res 2013;27:216-22.

30 Otsuji T, Abe M, Kinoshita H. After-effects of using a weighted bat on subsequent swing velocity and batters' perceptions of swing velocity and heaviness. Percept Mot Skills 2002;94:119-26.

31 Southard D, Groomer L. Warm-up with baseball bats of varying moments of inertia: effect on bat velocity and swing pattern. Res Q Exerc Sport 2003;74:270-6.

32 Torres EM, Anderson JM, Häkkinen K, et al. Effects of stretching on upper-body muscular performance. J Strength Cond Res 2008;22:1279-85.
33 Haag SJ, Wright GA, Gillette CM, et al. Effects of acute static stretching of the throwing shoulder on pitching performance of national collegiate athletic association division III baseball players. J Strength Cond Res 2010;24:452-7.

34 Knudson DV, Noffal GJ, Bahamonde RE, et al. Stretching has no effect on tennis serve performance. J Strength Cond Res 2004;18:654-6.

35 Franco BL, Signorelli GR, Trajano GS, et al. Acute effects of different stretching exercises on muscular endurance. J Strength Cond Res 2008; 22:1832-7.

36 Molacek ZD, Conley DS, Evetovich TK, et al. Effects of low- and high-volume stretching on bench press performance in collegiate football players. J Strength Cond Res 2010;24:711-16.

37 Cè E, Rampichini S, Maggioni MA, et al. Effects of passive stretching on post-activation potentiation and fibre conduction velocity of biceps brachii muscle. Sport Sci Health 2008;4:43-50.

38 Behm DG, Chaouachi A. A review of the acute effects of static and dynamic stretching on performance. Eur J App/ Physiol 2011;111:2633-51.

39 McHugh MP, Cosgrave CH. To stretch or not to stretch: the role of stretching in injury prevention and performance. Scand J Med Sci Sports 2010;20:169-81.

40 Sharman MJ, Cresswell AG, Riek S. Proprioceptive neuromuscular facilitation stretching: mechanisms and clinical implications. Cham: Adis International, 2006:929-39.

41 Khamwong P, Nosaka K, Pirunsan U, et al. Prophylactic effect of hot pack on symptoms of eccentric exercise-induced muscle damage of the wrist extensors. Eur J Sport Sci 2012;12:443-53.

42 Symons TB, Clasey JL, Gater DR, et al. Effects of deep heat as a preventative mechanism on delayed onset muscle soreness. J Strength Cond Res 2004:18:155-61.

43 Nosaka K, Sakamoto K, Newton M, et al. Influence of pre-exercise muscle temperature on responses to eccentric exercise. J Athl Train 2004;39:132-7.

44 Sedgwick $A$, Whalen $\mathrm{H}$. Effect of passive warm-up on muscular strength and endurance. Res Q Am Association Health Phys Educ Recreation 1964;35:45-59.

45 Surgeons AAoO. 10 Common Orthopaedic Injuries, 2014.

46 Freckleton $\mathrm{G}$, Pizzari T. Risk factors for hamstring muscle strain injury in sport: a systematic review and meta-analysis. Br J Sports Med 2013;47:351-8.

47 Opar MDA, Williams MD, Shield AJ. Hamstring strain injuries. Sports Med 2012:42:209-26. 